\title{
ON WEAKLY COMPACT SUBSETS OF BANACH SPACES
}

\section{H. H. $\operatorname{CORSON}^{1}$ AND J. LINDENSTRAUSS ${ }^{2}$}

Introduction. The two sections of this note are independent, but they are related by the fact that both use the results of [5] to obtain information on the properties of weakly compact sets in Banach spaces.

In the first section we prove some results on a class of compact sets which is believed to include all weakly compact subsets of Banach spaces. We are interested in the properties of the nonmetrizable sets of this form. (Our results become trivial in the metrizable case.) We show in particular that such sets have a dense subset consisting of $G_{\delta}$ points. Weakly compact sets are known to possess many properties which are similar to those of metrizable spaces (Eberlein's Theorem, for example). Our result exhibits a new property of this kind.

In the second section we show that in a separable reflexive space, every weakly compact set is the intersection of finite unions of cells.

1. Compact subsets of $C_{0}(\Gamma)$. Let $\Gamma$ be a discrete set and let $C_{0}(\Gamma)$ be the space of real-valued functions on $\Gamma$ which vanish at $\infty$. (A function $f$ from $\Gamma$ to the reals $R$ is said to vanish at $\infty$ if $\{\gamma ;|f(\gamma)|>\epsilon\}$ is finite for every $\epsilon>0$.) In $C_{0}(\Gamma)$ we take the topology of pointwise convergence. We can consider $C_{0}(\Gamma)$ also as a Banach space by taking the usual sup norm. The topology of pointwise convergence is weaker than the $w$-topology of the Banach space $C_{0}(\Gamma)$. A compact subset $K$ of $C_{0}(\Gamma)$ need not be norm bounded, and therefore it might fail to be compact in the w-topology. However, it is easily seen that such a set $K$ is linearly homeomorphic to a $w$-compact subset of $C_{0}(\Gamma)$. (Take a homeomorphism $\phi$ of the form $\phi f(\gamma)=\lambda_{\gamma} f(\gamma), f \in C_{0}(\Gamma), \gamma \in \Gamma$, where the $\lambda_{\gamma}$ are suitable positive numbers.)

We conjecture that the compact subsets of $C_{0}(\Gamma)$ are not only examples of weakly compact subsets of a Banach space but that every weakly compact subset of a Banach space is homeomorphic (perhaps even linearly homeomorphic) to a subset of $C_{0}(\Gamma)$ for some suitable $\Gamma$. It is clear that every metrizable weakly compact subset of a Banach space is linearly homeomorphic to a subset of $C_{0}(N)(N=$ the integers). Moreover, if there exists a bounded linear operator $T$ which is one to one from a Banach space $X$ into some $C_{0}(\Gamma)$, then $T$ maps the weakly compact subsets of $X$ homeomorphically on to com-

Received by the editors June 14, 1965.

1 Research fellow of the Alfred P. Sloan Foundation.

2 Research supported in part by the National Science Foundation (NSF GP-378). 
pact subsets of $C_{0}(\Gamma)$. For a large class of Banach spaces $X$ it is known that there exists an operator $T$ having the properties described above. For example, this is the case if $X=L_{p}(\mu)$, when $1 \leqq p<\infty$ and $\mu$ is an arbitrary measure, or if $p=\infty$ and $\mu$ is a $\sigma$-finite measure (cf. [1] for details). If $\mu$ is not $\sigma$-finite then $L_{\infty}(\mu)$ cannot be mapped continuously and linearly in a one to one manner into some $C_{0}(\Gamma)$ (this follows from the results of Day [3]), but we conjecture that on w-compact subsets of $L_{\infty}(\mu)$, which are in a sense small sets, such a mapping always exists. In this connection see also [7].

The compact subsets of $C_{0}(\Gamma)$ arise naturally in many problems, and in [1] and [2] our main results deal with some properties of these sets. Our first theorem here is applied in [2] for extending some results, which were known to be true for metrizable compact sets, to the more general class of compact subsets of $C_{0}(\Gamma)$.

In the statement of Theorem 1 we use the following notation. Let $K$ be a compact Hausdorff space. The space of real-valued continuous functions on $K$ with the sup norm is denoted by $C(K)$. The unit cell $\{\mu ;\|\mu\| \leqq 1\}$ of the dual $C(K)^{*}$ of $C(K)$ is denoted by $\Sigma(K)$.

Theorem 1. Let $K$ be a compact Hausdorff space. The set $\Sigma(K)$, taken in the w*-topology, is linearly homeomorphic to a subset of $C_{0}(\Gamma)$ for some set $\Gamma$ if and only if $K$ is homeomorphic to a subset of $C_{0}(\Gamma)$ for some $\Gamma$.

Proof. As is well known, $K$ is homeomorphic to a subset of $\Sigma(K)$, and therefore the "only if" part of the theorem is trivial.

Now let $K$ be a compact subset of $C_{0}(\Gamma)$ for some discrete space $\Gamma$. Without loss of generality we may assume that $|k(\gamma)| \leqq 1 / 2$ for every $k \in K$ and $\gamma \in \Gamma$. (If $K$ does not already satisfy this, we replace $K$ by $\phi K$ where $\phi$ is a homeomorphism of the type described in the beginning of the section.)

Let $\Omega$ be the set of all finite subsets of $\Gamma$. To each $\alpha=\left\{\gamma_{1}, \gamma_{2}, \cdots, \gamma_{n}\right\}$ in $\Omega$ let $f_{\alpha}$ be the element of $C(K)$ defined by $f_{\alpha}(k)=k\left(\gamma_{1}\right) k\left(\gamma_{2}\right) \cdots$ $k\left(\gamma_{n}\right)$. (If $\alpha=\varnothing$ then $f_{\alpha}(k)=1$ for every $k$.) It is easy to check that, for every fixed $k \in K$ and every $\epsilon>0$, the number of $\alpha \in \Omega$ such that $\left|f_{\alpha}(k)\right|>\epsilon$ is finite. Thus, for every sequence of distinct elements $\alpha_{1}, \alpha_{2}, \cdots$ in $\Omega$ and every $k \in K, \lim _{i \rightarrow \infty} f_{\alpha_{i}}(k)=0$. Since the $f_{\alpha}$ are uniformly bounded (by 1 ), we get that, for every finite measure $\mu$ on $K, \int_{K} f_{\alpha_{i}}(k) d \mu(k) \rightarrow 0$ as $i \rightarrow \infty$. Consequently, we can define a map $T: \Sigma(K) \rightarrow C_{0}(\Omega)$ by

$$
T(\mu)(\alpha)=\mu\left(f_{\alpha}\right)=\int_{K} f_{\alpha}(k) d \mu(k), \quad \alpha \in \Omega .
$$


Clearly $T$ is a continuous affine map. That $T$ is one to one follows from the fact that the linear combinations of the $f_{\alpha}, \alpha \in \Omega$, are norm dense in $C(K)$ (by the Stone-Weierstrass Theorem). Since $\Sigma(K)$ is $w^{*}$-compact, it follows that $T$ is a homeomorphism.

Corollary. Let $K$ be a compact subset of $C_{0}(\Gamma)$ for some set $\Gamma$. Then the Banach space $C(K)$ is isomorphic to a smooth Banach space. ( $A$ Banach space is called smooth if, for every $x \in X$ with $\|x\|=1$, there is exactly one $x^{*} \in X^{*}$ such that $\left\|x^{*}\right\|=x^{*}(x)=1$.)

Proof. Let $T$ be the map from $C(K)$ * into $C_{0}(\Omega)$ constructed in the proof of Theorem 1. In [3] Day constructed a norm $\||\cdot|\|$ in $C_{0}(\Omega)$ which is equivalent to the sup norm of $C_{0}(\Omega)$ and in which $C_{0}(\Omega)$ is strictly convex. Define a norm $\|\mid \cdot\| \|_{0}$ in $C(K)^{*}$ by \|\|$\mu\|\|_{0}=\||| T \mu\| \|$ $+\|\mu\|$ where $\|\cdot\|$ is the usual norm in $C(K)^{*}$. It is easily checked that the set $\left\{\mu ;\left|\|\mu \mid\|_{0} \leqq 1\right\}\right.$ is $w^{*}$-compact. Hence, by standard duality arguments (cf. [3]), the norm

$$
\|\mid f\|_{1}=\sup \left\{|\mu(f)| ;\|\| \mu \mid \|_{0} \leqq 1\right\}, \quad f \in C(K),
$$

is equivalent to the sup norm, and $C(K)$ with the norm \|\|$\cdot\|\|_{1}$ is a smooth Banach space.

Another result which can be proved by using Theorem 1(as well as results from [3] and [5]) is

Theorem 2. Let $K$ be a compact subset of $C_{0}(\Gamma)$. Then the $G_{\delta}$ points of $K$ form a dense subset of $K$.

Before proving Theorem 2, it is useful to collect some pertinent facts. A point $x \in K$ is called a $G_{\delta}$ point if it is the intersection of a sequence of open subsets of $K$. It is well known and easily checked that a point $x$ in a compact space is a $G_{\delta}$ point iff $x$ has a countable base of neighborhoods. Another simple and well known characterization of $G_{\delta}$ points (in a normal space) is the following. A point $x \in K$ is a $G_{\delta}$ point iff there exists a continuous real-valued function $f$ on $K$ such that $f(x)=1$ and $f(y)<1$ if $y \neq x$. Thus, in particular, every exposed point of a compact convex set $K$ in a linear topological space is a $G_{\delta}$ point. (A point $x \in K$ is called an exposed point if there exists a continuous linear functional $f$ on the space such that $f(x)=1$ and $f(y)<1$ if $x \neq y \in K$.) For the proof of Theorem 2 we need the following Lemma.

Lemma 1. $A$ w-compact convex subset $K$ of $C_{0}(\Gamma)$ is the closed convex hull of its exposed points.

Proof of Lemma 1. The proof of Theorem 1 of [5] shows that, if $K$ 
is a weakly compact convex subset of a Banach space $X$ and if $Y$ is another Banach space, then the set $P(K, Y)=\left\{T ;\|T x\|=\sup _{u \in K}\|T u\|\right.$ for some $x \in K\}$ is a dense subset of the Banach space $B(X, Y)=$ the space of all bounded linear operators from $X$ to $Y$ with the usual operator norm $\left(\|T\|=\sup _{\| x \rrbracket \leq 1}\|T x\|\right)$.

The proof of Theorem 2 of [5] shows that, if

(1) $K$ is a symmetric convex closed subset of a Banach space $X$,

(2) $X$ is isomorphic to a strictly convex Banach space $Y$,

(3) $P(K, Y)$ is norm dense in $B(X, Y)$,

then $K$ is the closed convex hull of its exposed points.

Since $C_{0}(\Gamma)$ is isomorphic to a strictly convex space (cf. [3]), it follows that every symmetric w-compact convex subset of $C_{0}(\Gamma)$ is the closed convex hull of its exposed points.

If $K$ is a nonsymmetric compact convex subset of $C_{0}(\Gamma)$, consider the subset $K_{0} \subset C_{0}(\Gamma) \oplus R$ which is the convex hull of

$$
\{(k, 1) ; k \in K\} \cup\{(-k,-1) ; k \in K\} .
$$

We have already proved that $K_{0}$ is the closed convex hull of its exposed points. The fact that the same is true for $K$ is an easy consequence.

Proof of Theorem 2. By Theorem 1 and Lemma 1 the set $\Sigma(K)$ is the $w^{*}$-closed convex hull of its exposed points. By a theorem of Milman [4, p. 80] it follows that the exposed points of $\Sigma(K)$ are dense in the extreme points of $\Sigma(K)$. The extreme points of $\Sigma(K)$ are of the form $\pm \psi_{x}$ where $\psi_{x}$ is the evaluation at $x\left(\psi_{x}(f)=f(x), f \in C(K)\right.$, $x \in K)$. It is clear that $\psi_{x}$ is an exposed point of $\Sigma(K)$ iff $x$ is a $G_{\delta}$ point of $K$. This concludes the proof.

REMARKs. 1. An extreme point of a convex w-compact set $K$ in a Banach space need not be a $G_{\delta}$ point of $K$ in the $w$-topology. Take for example as $K$ the positive part of the unit cell of $l_{2}(\Gamma)$ with $\Gamma$ uncountable: $K=\left\{x \in l_{2}(\Gamma) ; x(\gamma) \geqq 0\right.$ for every $\gamma$ and $\left.\Sigma_{\gamma} x^{2}(\gamma) \leqq 1\right\}$. The origin is an extreme point of $K$ but not a $G_{\delta}$ point.

2. There exist non-metrizable compact subsets $K$ of $C_{0}(\Gamma)$ which are first countable (i.e. every point of $K$ is a $G_{\delta}$ point). Take for example $\Gamma=[0,1]$, and let $K$ be the set consisting of the points $x_{\gamma}$, $\gamma \in[0,1]$, and $y_{\gamma}, \gamma \in[0,1]$, where the $x_{\gamma}$ and $y_{\gamma}$ are defined by

$$
\begin{array}{ll}
x_{\gamma}(0)=\gamma, & x_{\gamma}(\alpha)=0, \quad \text { if } \alpha \neq 0, \\
y_{\gamma}(0)=\gamma, & y_{\gamma}(\gamma)=1, \quad y_{\gamma}(\alpha)=0, \quad \text { if } \alpha \neq 0, \gamma .
\end{array}
$$

2. Representation of $w$-compact subsets of separable reflexive spaces. The theorem we prove in this section is somewhat related to the work of Phelps [6]. 
Theorem 3. Let $X$ be a separable reflexive Banach space. A subset $K$ of $X$ is w-compact if and only if it is the intersection of finite unions of cells.

The proof of this theorem is based on the following result [5, Theorem $4 \mathrm{~b}]$.

Lemma 2. Let $X$ be as in Theorem 3, then the strongly smooth points are norm dense in the boundary of the unit cell of $X$. ( $A$ point $x$ with $\|x\|=1$ is called strongly smooth if there is an $x^{*} \in X^{*}$ with $\left\|x^{*}\right\|$ $=x^{*}(x)=1$ such that $\|x+y\|=1+x^{*}(y)+o(\|y\|)$ as $\|y\| \rightarrow 0$.)

Proof of Theorem 3. In this proof we shall denote the cell $\left\{x ;\left\|x-x_{0}\right\| \leqq r\right\}$ by $S\left(x_{0}, r\right)$. Since every cell in a reflexive space is $w$-compact, the "if" part of the theorem is trivial. Hence it is enough to prove that every w-closed subset of $S(0,1)$ is the intersection of finite unions of cells. Let $\tau$ be the topology on $S(0,1)$ in which the closed sets are exactly the intersections of finite unions of cells. The topology $\tau$ is weaker than the w-topology. Since $S(0,1)$ is w-compact, our theorem will be proved once it is shown that $\tau$ is a Hausdorff topology.

We shall prove the following assertion which is slightly stronger than the assertion that $\tau$ is a Hausdorff topology.

(\#) Let $y_{1} \neq y_{2}$ be two points in $S(0,1)$. Then there exist two cells $S\left(x_{1}, r_{1}\right)$ and $S\left(x_{2}, r_{2}\right)$ in $X$ such that $y_{i} \notin S\left(x_{i}, r_{i}\right), i=1,2$, and such that

$$
S\left(x_{1}, r_{1}\right) \cup S\left(x_{2}, r_{2}\right) \supset S(0,1) .
$$

Let $z=y_{1}-y_{2}$ and let $u$ be a strongly smooth point of $S(0,1)$ with

$$
\|u-z /\| z\|\|<\frac{1}{6}, \quad\|u\|=1
$$

(cf. Lemma 2). Now put

$x_{1}^{n}=y_{2}-(n-2\|z\| / 3) u, \quad x_{2}^{n}=y_{1}+(n-2\|z\| / 3) u, \quad n=1,2, \cdots$

For every $n$

$$
\begin{aligned}
\left\|x_{1}^{n}-y_{1}\right\| & =\|z+(n-2\|z\| / 3) u\| \\
& \geqq\|(n+\|z\| / 3) u\|-\|z\|\|u-z /\| z\|\|>n .
\end{aligned}
$$

Similarly, $\left\|x_{1}^{n}-y_{2}\right\|>n$ for every $n$. Hence, in order to prove (\#) we have only to show that for some integer $n$,

$$
S\left(x_{1}^{n}, n\right) \cup S\left(x_{2}^{n}, n\right) \supset S(0,1) \text {. }
$$


Suppose that (2) is false. Then for every $n$ there is a $z^{n} \in S(0,1)$ such that

$\left\|z^{n}-y_{2}+(n-2\|z\| / 3) u\right\| \geqq n$ and $\left\|z^{n}-y_{1}-(n-2\|z\| / 3) u\right\| \geqq n$, or equivalently

$$
\left\|u+\left(z^{n}-y_{2}-2\|z\| u / 3\right) / n\right\| \geqq 1 \text { and }\left\|u+\left(y_{1}-z^{n}-2\|z\| u / 3\right) / n\right\| \geqq 1 .
$$

Since $u$ is a strongly smooth point, there is a $u^{*} \in X^{*}$ with $u^{*}(u)$ $=\left\|u^{*}\right\|=1$ and such that as $n \rightarrow \infty$

$$
u^{*}\left(z^{n}-y_{2}-2\|z\| u / 3\right) \geqq o(1) \quad \text { and } \quad u^{*}\left(y_{1}-z^{n}-2\|z\| u / 3\right) \geqq o(1) \text {. }
$$

Adding these two inequalities we get that

$$
0 \leqq u^{*}\left(y_{1}-y_{2}-4\|z\| u / 3\right)=u^{*}(z)-4\|z\| u^{*}(u) / 3 .
$$

On the other hand we have that $u^{*}(z) \leqq\|z\|$ and this contradicts (3). We have thus proved that (2) holds, and this concludes the proof of the theorem.

REMARKS. 1. It is not true in general that a set in a conjugate separable Banach space is $w^{*}$-compact if and only if it is the intersection of finite unions of cells. For example the subset $K$ of $l_{1}=c_{0}^{*}$ defined by $K=\left\{x \in l_{1} ; x(1) \geqq 0,\|x\| \leqq 1\right\}$ is $w^{*}$-compact, but it cannot be represented as the intersection of finite union of cells. In fact, it is easily verified that every finite union of cells in $l_{1}$ which contains the set $K$ already contains the whole unit cell of $l_{1}$.

2. We do not know whether Theorem 3 will hold if we drop the assumption that $X$ is separable.

\section{REFERENCES}

1. H. H. Corson and J. Lindenstrauss, On function spaces which are Lindelöf spaces, Trans. Amer. Math. Soc. 121 (1966), 476-491.

2. - Continuous selections with nonmetrizable range, Trans. Amer. Math. Soc. 121 (1966), 492-504.

3. M. M. Day, Strict convexity and smoothness of normed spaces, Trans. Amer. Math. Soc. 78 (1955), 516-528.

4. - Normed linear spaces, Springer, Berlin, 1958.

5. J. Lindenstrauss, On operators which attain their norm, Israel J. Math. 1 (1963), 139-148.

6. R. R. Phelps, A representation theorem for bounded convex sets, Proc. Amer. Math. Soc. 11 (1960), 976-983.

7. J. Lindenstrauss, On reflexive spaces having the metric approximation property, Israel J. Math. (to appear).

UNIVERSITY OF WASHINGTON 